\title{
Time evolution in the Morse potential using supersymmetry: dissociation of the NO molecule
}

\author{
Balázs Molnár, Péter Földi, Mihály G. Benedict,f and Ferenc Bartha \\ Department of Theoretical Physics, Unversity of Szeged, Tisza L. körút 84, H-6720 Szeged, Hungary
}

(Dated: October 26, 2018)

\begin{abstract}
We present an algebraic method for treating molecular vibrations in the Morse potential perturbed by an external laser field. By the help of a complete and normalizable basis we transform the Schrödinger equation into a system of coupled ordinary differential equations. We apply our method to calculate the dissociation probability of the NO molecule excited by chirped laser pulses. The dependence of the molecular dipole-moment on the interatomic separation is determined by a quantum-chemical method, and the corresponding transition dipole moments are given by approximate analytic expressions. These turn out to be very small between neighboring stationary states around the vibrational quantum number $m=42$, therefore we propose to use additional pulses in order to skip this trapping state, and to obtain a reasonable dissociation probability.
\end{abstract}

PACS numbers: 33.80.-b, 3.65.-w

An important model for anharmonic molecular vibrations, allowing also for dissociation is the Morse Hamiltonian [1]

$$
\widehat{H}_{0}=\frac{\widehat{P}^{2}}{2 m}+D_{0}[\exp (-2 \alpha \widehat{X})-2 \exp (-\alpha \widehat{X})] .
$$

We shall consider the time development of a molecular state in this potential coupled also to an external classical field via the dipole interaction. Thus we shall give the solution of the time dependent Schrödinger equation:

$$
i \hbar \frac{d|\Phi\rangle}{d t}=\left[\widehat{H}_{0}-\widehat{\mu} E(t)\right]|\Phi\rangle
$$

where $\widehat{\mu}(\widehat{X})$ is the molecular dipole moment, and $E$ is the electric field strength of the external field. Introducing the parameters: $\omega_{0}=\alpha \sqrt{\frac{2 D_{0}}{m}}$ and $s=\frac{\sqrt{2 m D_{0}}}{\hbar \alpha}-1 / 2$, and the dimensionless operators $X=\alpha \widehat{X}, P=\widehat{P} / \alpha \hbar$ the dimensionless Hamiltonian $H_{0}=\left\{(2 s+1) / \hbar \omega_{0}\right\} \widehat{H}_{0}$ can be recast in the form

$$
H_{0}=A^{\dagger}(s) A(s)-s^{2} .
$$

Here $A(q)=q I-(s+1 / 2) \exp (-X)+i P$, and $A^{\dagger}(q)=$ $q I-(s+1 / 2) \exp (-X)-i P$ are generalized supersymmetric ladder operators [2], allowing the case $q \neq s$, as well. They satisfy the following commutation relations

$$
\begin{aligned}
{\left[A(q), A^{\dagger}\left(q^{\prime}\right)\right] } & =\left(q+q^{\prime}\right) I-\left(A(q)+A^{\dagger}\left(q^{\prime}\right)\right), \\
{\left[A(q), A\left(q^{\prime}\right)\right] } & =0, \quad\left[A^{\dagger}(q), A^{\dagger}\left(q^{\prime}\right)\right]=0 .
\end{aligned}
$$

As $A^{\dagger}(s) A(s)=A(s) A^{\dagger}(s)-2 s+1$ is a shape invariant supersymmetric operator [2], the solution of the eigenvalue equation $H_{0}\left|\psi_{m}(s)\right\rangle=E_{m}(s)\left|\psi_{m}(s)\right\rangle$ can be found as follows [3]

$$
\begin{aligned}
\left|\psi_{m}(s)\right\rangle & =\mathcal{N}_{m} A^{\dagger}(s) \cdots A^{\dagger}(s-m+1)\left|\psi_{0}(s-m)\right\rangle, \\
E_{m}(s) & =-(s-m)^{2}, \quad m=0,1, \ldots[s],
\end{aligned}
$$

where $\mathcal{N}_{m}=\sqrt{\frac{(2 s-2 m) !}{m !(2 s-m) !}}$, and $\left|\psi_{0}(s-m)\right\rangle$ is the single nondegenerate vector annihilated by $A(s-m)$

$$
A(s-m)\left|\psi_{0}(s-m)\right\rangle=0 .
$$

Using coordinate representation it can be simply proven that $\left|\psi_{0}(s-m)\right\rangle$, as well as $\left|\psi_{m}(s)\right\rangle$ are normalizable if and only if $m<s$, therefore the number of bound states is $[s]+1$, where $[s]$ denotes the largest integer that is smaller than $s$. The infinite dimensional Hilbert space of the problem is, however a direct sum: $\mathcal{H}=\mathcal{H}^{-} \oplus \mathcal{H}^{+}$, where $\mathcal{H}^{-}$is the finite $([s]+1)$ dimensional subspace spanned by the bound states, while $\mathcal{H}^{+}$is an infinite dimensional subspace with elements obtainable as continuous superpositions of positive energy eigenstates.

Instead of using the non-normalizable continuum energy eigenstates, we introduce algebraically a true orthonormal basis in $\mathcal{H}$ that allows a natural discretization of time dependent problems. Let $\sigma=s-[s]$, and starting from the state $\left|\phi_{0}\right\rangle$ defined by the relation $A(\sigma)\left|\phi_{0}\right\rangle=0$, we introduce the following series of states

$$
\left|\phi_{n}\right\rangle=\left\{\prod_{k=1}^{n} C_{k}^{-1} A^{\dagger}(\sigma+k-1)\right\}\left|\phi_{0}\right\rangle,
$$

where the coefficients $C_{k}=\sqrt{k(k+2 \sigma-1)}$ ensure the normalization. We will call the states $\left|\phi_{n}\right\rangle$ quasi-number states 清. From the definition (7) it is also seen that the neighboring quasi-number states are connected as

$$
\begin{aligned}
A^{\dagger}(\sigma+n)\left|\phi_{n}\right\rangle & =C_{n+1}\left|\phi_{n+1}\right\rangle, \\
A(\sigma+n)\left|\phi_{n}\right\rangle & =C_{n}\left|\phi_{n-1}\right\rangle .
\end{aligned}
$$

The orthogonality of the $\left|\phi_{n}\right\rangle$ states can be seen by using the commutators (14). In coordinate representation they have the form

$$
\phi_{n}(x)=\sqrt{n ! / \Gamma(2 \sigma+n)} y^{\sigma}(x) \exp \left(-\frac{y(x)}{2}\right) L_{n}^{2 \sigma-1}(y(x)),
$$


where $L_{n}^{2 \sigma-1}$ is a generalized Laguerre polynomial [5, 6] of the variable $y(x)=(2 s+1) e^{-x}$. In contrast to the energy eigenfunctions, these wave functions form a complete orthonormal set of square integrable functions [6], therefore the quasi-number states constitute a true orthonormal basis in the Hilbert space.

Using the properties of the supersymmetric ladder operators $A^{\dagger}$ and $A$, one can calculate the matrix of $H_{0}$ in this basis

$$
\begin{aligned}
& \left\langle\phi_{m}\left|H_{0}\right| \phi_{n}\right\rangle=\left(C_{m}^{2}-s^{2}+(m-[s])^{2}\right) \delta_{m, n} \\
& +([s]-n) C_{m} \delta_{m, n+1}+([s]-m) C_{n} \delta_{m+1, n}
\end{aligned}
$$

It consists of two blocks, the one corresponding to $0 \leq n, m \leq[s]$ is $[s]+1$ dimensional, whereas the other one, corresponding to $n, m>[s]$ is infinite dimensional, and both blocks are tridiagonal. The block structure shows that the first $[s]+1$ quasi-number states span an invariant subspace of $H_{0}$, in which it can be diagonalized. This subspace should coincide with $\mathcal{H}^{-}$, as $H_{0}$ has exactly $[s]+1$ nondegenerate eigenvalues with normalizable eigenstates. The orthogonality relation

$$
\left\langle\phi_{n} \mid \psi_{m}\right\rangle=0, \quad \text { for } m=0,1 \ldots[s], \text { and } n>[s]
$$

equivalent to the above statement can be proven directly, and the expressions of the overlaps $\left\langle\phi_{n} \mid \psi_{m}\right\rangle$ for $n \leq[s]$ can be determined explicily [7].

We will consider the time evolution of molecular vibrations subject to an external classical field as described by Eq. (2). In the dimensionless units used in Eq. (3) the interaction term, $\widehat{H}_{\text {int }}=-\widehat{\mu}(\hat{X}) E(t)$ can be written as

$$
H_{\mathrm{int}}=-\mu(X) \mathcal{E}(t),
$$

where we have introduced $\mu=\frac{\alpha}{q_{e}} \widehat{\mu}$, with an effective charge $q_{e}=\left.\frac{d \widehat{\mu}(x)}{d x}\right|_{x=0}$, and $\mathcal{E}(t)=\frac{(2 s+1) q_{e}}{\hbar \omega_{0} \alpha} E(t)$ denotes the electric field strength in our units. As we will solve the Schrödinger equation (2) in the quasi-number state basis, we also need to know the matrix of the dipole moment in this basis. Due to the algebraic properties of the quasi-number states, the matrix of $\mu(X)$ can also be determined analytically for certain cases. Let us suppose that the dipole moment can be approximated by an operator of the form

$$
\mu(X)=\sum_{i} \mu_{i}(X)=\sum_{i}\left(a_{i} X+d_{i}\right) e^{-\gamma_{i} X},
$$

where $a_{i}, d_{i}$ and $\gamma_{i}$ are real numbers. Taking only a single term with $d=\gamma=0$ would mean the simplest linear dipole moment borrowed from atomic calculations and used sometimes for molecules as well. This latter approach, however, overestimates the strength of the interaction at larger atomic separations and leads to an unrealistically high dissociation probability.

By the aid of Eq. (7) and the fact that $\left[\mu(X), A^{\dagger}(q)\right]=$ $[\mu(X),-i P]=\frac{d \mu(X)}{d X}$, recurrence relations can be derived for the matrix elements of $\mu(X)$ between the states $\left|\phi_{n}\right\rangle$. We have

$$
\begin{gathered}
\left\langle\phi_{m}\left|X e^{-\gamma X}\right| \phi_{n+1}\right\rangle=\left[(n-m-\gamma)\left\langle\phi_{m}\left|X e^{-\gamma X}\right| \phi_{n}\right\rangle\right. \\
\left.+C_{m}\left\langle\phi_{m-1}\left|X e^{-\gamma X}\right| \phi_{n}\right\rangle+\left\langle\phi_{m}\left|e^{-\gamma X}\right| \phi_{n}\right\rangle\right] / C_{n+1}, \\
\left\langle\phi_{m}\left|e^{-\gamma X}\right| \phi_{n+1}\right\rangle=\left[C_{m}\left\langle\phi_{m-1}\left|e^{-\gamma X}\right| \phi_{n}\right\rangle\right) \\
\left.+(n-m-\gamma)\left\langle\phi_{m}\left|e^{-\gamma X}\right| \phi_{n}\right\rangle\right] / C_{n+1} .
\end{gathered}
$$

Then all the necessary matrix elements can be calculated, starting from terms $\left\langle\phi_{0}|X \exp (-\gamma X)| \phi_{0}\right\rangle$ and $\left\langle\phi_{0}|\exp (-\gamma X)| \phi_{0}\right\rangle$. These latter can be obtained via integration in coordinate representation using the wave function $\phi_{0}(x)$ of Eq. (9), and we get

$$
\begin{gathered}
\left\langle\phi_{0}\left|X e^{-\gamma X}\right| \phi_{0}\right\rangle=\frac{\Gamma(2 \sigma+\gamma)}{(2 s+1)^{\gamma} \Gamma(2 \sigma)}[\ln (2 s+1)-\widetilde{\psi}(2 \sigma+\gamma)], \\
\left.\left\langle\phi_{0}\right| e^{-\gamma X}\right)\left|\phi_{0}\right\rangle=\frac{\Gamma(2 \sigma+\gamma)}{(2 s+1)^{\gamma} \Gamma(2 \sigma)},
\end{gathered}
$$

where $\widetilde{\psi}$ denotes Euler's digamma function [5].

The values of the parameters $a_{i}, d_{i}$ and $\gamma_{i}$ can be obtained by fitting the function (13) to experimental dipole curves, or to those obtained from molecular calculations.

The quasi-number states constitute a complete orthonormal basis, hence one can expand the solution $|\Phi(t)\rangle$ of Eq. (2) in the form: $|\Phi(t)\rangle=\sum_{n=0}^{\infty} c_{n}(t)\left|\phi_{n}\right\rangle$. Measuring the time $t$ in units of $T=2 \pi / \omega_{0}$, the Schrödinger equation can be written as the following infinite system of ordinary differential equations

$$
i \dot{c}_{n}=\frac{2 \pi}{2 s+1} \sum_{m=0}^{\infty}\left\{\left\langle\phi_{n}\left|H_{0}\right| \phi_{m}\right\rangle-\mathcal{E}(t)\left\langle\phi_{n}|\mu| \phi_{m}\right\rangle\right\} c_{m} .
$$

where $\left\langle\phi_{n}\left|H_{0}\right| \phi_{m}\right\rangle$ and $\left\langle\phi_{n}|\mu| \phi_{m}\right\rangle$ are given by the expressions (10) and (14), respectively. We note here that the usual method of solving the time evolution of the molecular state is based on the split operator method [8]. That approach solves the partial differential equation corresponding to Eq. (2), and yields an approximate wave function of the variables $x$ and $t$. In contrast, we solve a system of ordinary equations allowing a higher accuracy in the calculations.

We will apply our method to determine the dissociation probability of a molecule. This is a time dependent quantity defined as the projection probability of $|\Phi(t)\rangle$ on $\mathcal{H}^{+}$that can be given as

$$
\mathcal{P}(t)=1-\sum_{m=0}^{[s]}\left|\left\langle\Phi(t) \mid \psi_{m}\right\rangle\right|^{2}=1-\sum_{m=0}^{[s]} c_{m}^{*}(t) c_{m}(t) .
$$

To calculate the dissociation probability above, one has to solve the set of the dynamical equations (16) and determine the coefficients $c_{m}(t)$ for $0 \leq m \leq[s]$. In practice 
one has to truncate the system (16), and we have to use a numerical method to find the solution.

The restriction of the number of the dynamical equations to a finite number is done here in two steps. First we consider the problem in a large subspace of dimension $N \gg[s]$, and neglect the contribution of those states $\left|\phi_{n}\right\rangle$ for which $n>N$. Then the Hamiltonian is represented by an operator which is restricted to the finite $N$ dimensional subspace, spanned by the orthonormal system: $\left\{\left|\psi_{m}\right\rangle, m=0,1,2 \ldots[s], \quad\left|\phi_{n}\right\rangle, n=[s]+1, \ldots N\right\}$. We denote this operator by $H^{(N)}=H_{0}^{(N)}-\mu^{(N)} E(t)$ where $H_{0}^{(N)}$ and $\mu^{(N)} E(t)$ are the truncated operators, so that their matrices are of dimension $N$.

Before solving the already finite number of ordinary differential equations, we perform a unitary transformation on our truncated basis and bring $H_{0}^{(N)}$ into diagonal form for $n>[s]$, too. Due to the fact that the matrix of $H_{0}^{(N)}$ is finite tridiagonal this can be done by the help of a very fast algorithm. This yields positive energy eigenvalues $E_{n}^{(N)}([s]<n \leq N)$ and eigenstates $\left|\psi_{n}^{N}\right\rangle$, which we can use for the description of the dissociated molecule. If $N$ is large enough, then the lowermost positive energies $E_{n}^{(N)}$ follow densely each other, and approximate satisfactorily the continuous energy spectrum above the dissociation threshold. In the second step of the approximation, we restrict the calculation to the bound subspace and to those $\left|\psi_{n}^{N}\right\rangle$ states with $n>[s]$, for which the dipole couplings with the bound energy eigenstates are non-negligible. In the application to be discussed below, it turns out that the latter are significant only for those states that correspond to the lowest positive energy values $E_{n}^{(N)}$ with $n=[s]+1, \ldots M$, so that $M \ll N$. Therefore, in order to follow the dissociation process, it is enough to solve Eq. (16) in the basis $\left|\psi_{m}\right\rangle,\left|\psi_{n}^{N}\right\rangle$, which means that we expand the time dependent state as $|\Phi(t)\rangle=\sum_{n=0}^{M} b_{n}(t)\left|\psi_{n}\right\rangle$, where now the notation $\left|\psi_{n}\right\rangle$ has been used also for $\left|\psi_{n}^{N}\right\rangle$ with $n=[s]+1, \ldots M$. Then we solve the system of $M$ ordinary differential equations that describes the time evolution of the coefficients $b_{n}$ and calculate the dissociation probability via Eq. (17) with the obvious $c_{m}(t) \rightarrow b_{m}(t)$ replacement.

In what follows we are going to apply our method for the determination of the dissociation probability of the nitrogen-oxide (NO) molecule, excited by an appropriate laser field [9]. We have used a Morse potential with the following data in the electronic ground state [10: $m=7.46$ a.u., $D=6.497 \mathrm{eV}$ and $\alpha=27.68 \mathrm{~nm}^{-1}$, yielding $s=54.54$. The potential and the dependence of the dipole moment on the interatomic separation are depicted in Fig. 1. The circles show the dipole moments of $\mathrm{NO}$ at various interatomic separations that we have calculated by an unrestricted density functional method [11. The dotted line is a fitted function of the form Eq. (13) with two terms, and with the parameter values: $a_{1}=-9.66, a_{2}=10.64, d_{1}=d_{2}=0, \gamma_{1}=0.927$,

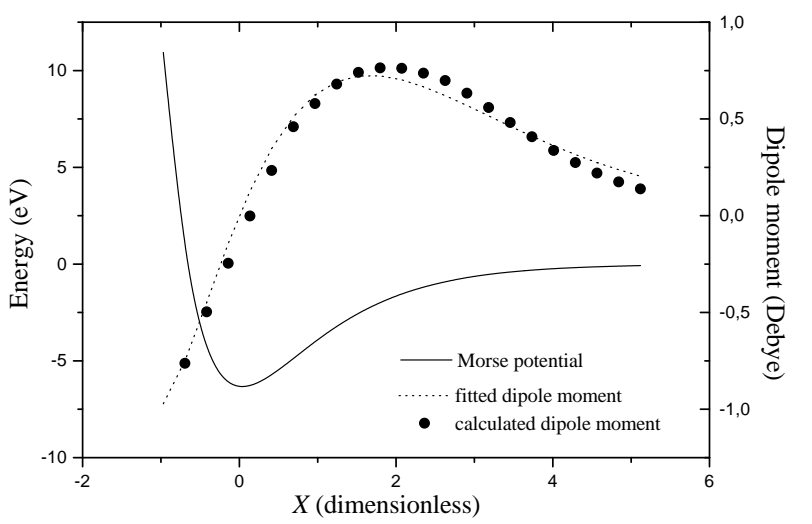

FIG. 1: The Morse potential and the dipole moment of the NO molecule.

$\gamma_{2}=0.870$

The corresponding matrix elements have been determined analytically by using the recurrence relations (14. 15), and those between the first second and third neighbors are shown in Fig 2. In our calculations we have

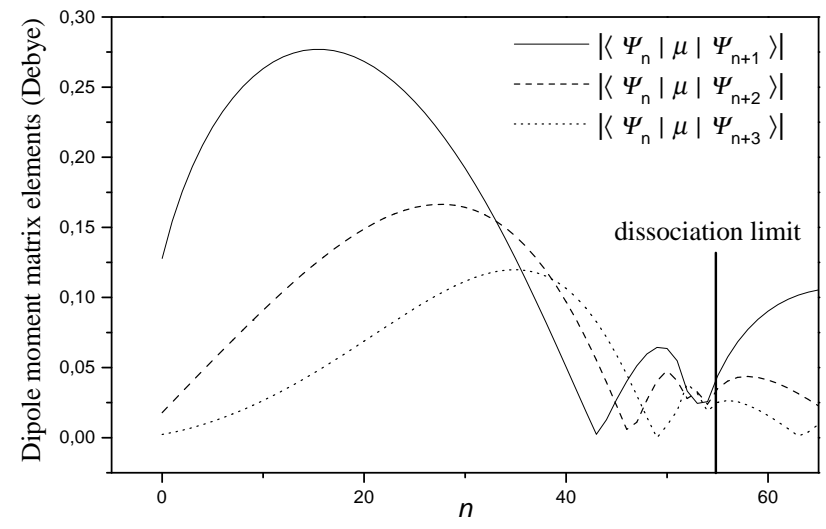

FIG. 2: Absolute values of the dipole matrix elements between first, second, and third neighbor states. The matrix elements far from the diagonal are diminishingly small.

chosen the truncation indices to be $N=3000$, and $M=200$, as increasing these values had no effect on the results. (Note that the number of bound states is now $[s]+1=55)$. The initial state was taken to be the vibrational ground state, and we devised the excitation with the aim to acheive a significant dissociation probability.

According to 12 one can think of the desired time evolution as a sequence of two-level transitions. There are two conditions to be fulfilled: the electric field should be approximately resonant with the actual transition, and its area per transition [12] should be around $\pi$. In order to maintain the resonance condition during the whole process, it is appropriate to use continuously chirped pulses 12. If the frequency of the pulse decreases slowly enough, then it is possible to consider its effect as a sequence of approximately resonant two-level transitions. 
For low vibrational quantum numbers the coupling is largest between the nearest neighbors, therefore at the beginning the excitation has to drive the system through the ladder of neighboring energy eigenstates towards the continuum. However, as it is seen in Fig. 2, there is a minimum in the $m \rightarrow m+1$ couplings at $m=42$, therefore this vibrational quantum number corresponds to a trapping state. This is a consequence of the shape of the dipole moment curve (Fig. 11) and it would be absent in the linear approximation $\mu(X) \sim X$. One can circumvent the problem by lengthening the pulse in time, but we present a different route here with the three pulses shown in Fig. 3 exploiting other than first neighbor transitions, as well. The area condition could be met for all

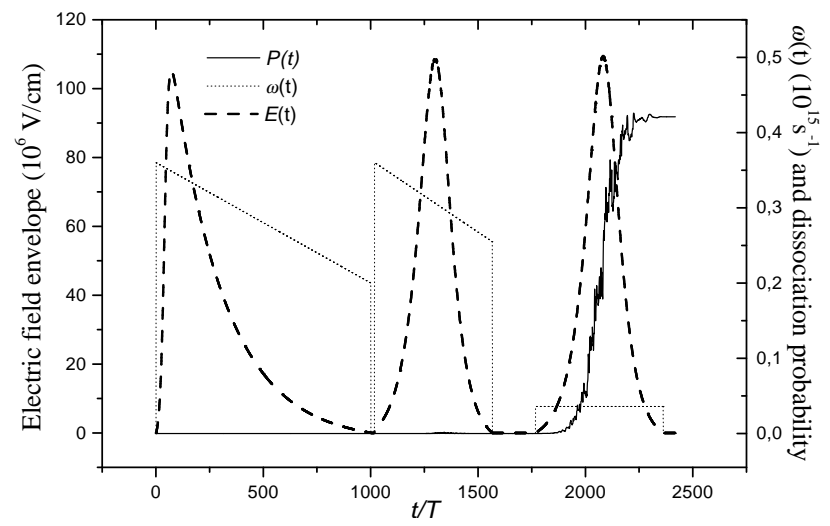

FIG. 3: Electric field strength envelopes and time dependent frequencies of the applied pulses. Dissociation probability is also shown as a function of time measured in units of $T=$ $2 \pi / \omega_{0}$. All three pulses have a distorted secant-hyperbolic envelope (see text) with the data: pulse (1): $\tau_{f}=10.27$, $\tau_{t}=246.4, t_{0}=41.0 ;$ pulse $(2): \tau_{f}=71.87, \tau_{t}=61.61$, $t_{0}=1297.5$; pulse $(3): \tau_{f}=71.87, \tau_{t}=61.61, t_{0}=2087.6$. Peak values for all three pulses are: $1.05 \times 10^{8} \mathrm{~V} / \mathrm{cm}$.

three pulses with distorted secant-hyperbolic envelopes: $\mathcal{E}=\mathcal{E}_{a} /\left[\exp \left(t_{0}-t\right) / \tau_{f}+\exp \left(t-t_{0}\right) / \tau_{t}\right]$ with different exponentials in their fronts $\left(\tau_{f}\right)$, and tails $\left(\tau_{t}\right)$. The chirping rates are seen in Fig. 2, while the other data of the pulses are given in the caption. Using these parameters, the first pulse drives the molecule into a superposition of a few states $\left|\psi_{m}\right\rangle$ around $m=31$. As seen in Fig. 2, the $m \rightarrow m+2$ matrix elements are getting larger here than those connecting the neighboring states, and the former have also nonvanishing values around $m=42$. This implies that a second laser pulse with an initial carrier frequency being in approximate resonance with the corresponding $m \rightarrow m+2$ transition will continue the dissociation process and let the molecular state jump over the trap. After this second pulse, the distribution of the populated states is not so narrow as it was before, which is a consequence of the presence of second neighbor transitions. The most probably populated states are now so close to the continuum limit that a final pulse - even without chirping - leads to a dissociation probability that is more than $40 \%$. As shown in Fig. 3, this probability is practically zero before the third pulse.

In summary, we have presented a method based on supersymmetric quantum mechanics that leads to a system of ordinary differential equations describing molecular time evolution. We have applied this method for devising laser pulses leading to a significant dissociation probability of the NO molecule with a realistic dipole moment.

We thank A. Czirják and A. Lörincz for discussions. This work was supported by the Hungarian Scientific Research Fund (OTKA) under contract No. T32920, and by the Hungarian Ministry of Education under contract No. FKFP 099/2001.

* Electronic address: benedict@physx.u-szeged.hu

[1] P. M. Morse, Phys. Rev. 34, 57, (1929), L. D. Landau and E. M. Lifshitz, Quantum Mechanics, Nonrelativistic Theory, (Pergamon Press, Oxford, 1965).

[2] L. E. Gendenshtein Pisma Zh. Eksp Teor. Fiz., 38, 299 (1983), [JETP Lett. 38, 356 (1983)], F.Cooper, A. Khare, and U.P. Shukhatme Phys. Rep. 251, 268 (1995).

[3] M. G. Benedict and B. Molnár, Phys. Rev. A 60, R1737 (1999).

[4] A somewhat different set of basis functions called pseudonumber states were introduced in Ref. 3 in the construction of the coherent states of the Morse potential. Time evolution in terms of pseudo-number states was considered in Ref. 13, while the algebraic background has been discussed in Ref. 14.

[5] M. Abramowitz and I. Stegun, Handbook of Mathematical Functions (Dover, 1965).

[6] G. Szegő, Orthogonal Polynomials, (Am. Math. Soc., New York, 1959).

[7] These calculations use the properties of the supersymmetric ladder operators, and will be published elsewhere.

[8] M. Feit, J. A. Fleck Jr., A. Steiger, J. Comput Phys. 47, 412 (1982). For a detailed review of the availabe methods see R. Kosloff, J. Phys. Chem. 92, 2087 (1988).

[9] For calculations with other methods see: J. Ting, J. Phys. B: At. Mol. Opt. 27 1249(1994), J-M. Yuan, W-K. Liu, Phys. Rev. A 57, 1992 (1998).

[10] K. P. Huber and G. Herzberg, Molecular spectra and molecular structure IV. Constants of diatomic molecules, (van Nostrand Reinhold, 1979).

[11] We have used the Gaussian 98 program package with the B3LYP functional and the 6-311G(3d2f) basis set.

[12] Sz. Chelkowski A. D. Bandaruk, and P. B. Corkum, Phys.Rev. Lett. 65, 2355 (1990), Sz. Chelkowski and G. N. Gibson, Phys. Rev. A 52, R3417 (1995).

[13] B. Molnár, M. G. Benedict, and P. Földi, Fortsch. Phys. 49, 1053 (2001).

[14] B. Molnár, M. G. Benedict, and J. Bertrand J. Phys A: Math. Gen. 34, 3139 (2001). 